\title{
Multi-Anode Sawtooth SDD for X-Ray Spectroscopy Fabricated on NTD Wafers
}

\author{
J. Šonský, R. W. Hollander, Member, IEEE, C. W. E. van Eijk, Member, IEEE, P. M. Sarro, and V. Kouchpil
}

\begin{abstract}
We are developing a multi-anode sawtooth silicon drift detector (MSSDD) with an anode pitch of $250 \mu \mathrm{m}$ for one-dimensional position-sensitive detection of low-energy X-rays down to $\sim 200 \mathrm{eV}$. The detector is intended to be used in X-ray diffraction analysis. In this paper, we present new results of $X$-ray spectroscopy measurements with detectors fabricated on neutron transmutation doped (NTD) wafers with a thickness of $290 \mu \mathrm{m}$. Using an MSSDD with an anode pitch of $250 \mu \mathrm{m}$ and having $\mathrm{p}^{+}$ strips on both sides, we have measured an energy resolution of 191-eV full-width half-maximum (FWHM) per anode pixel for the $5.89 \mathrm{keV}$ line of ${ }^{55} \mathrm{Fe}$ at $213 \mathrm{~K}$. At room temperature the energy resolution is $375 \mathrm{eV}$ FWHM. Split events are almost completely eliminated due to the sawtooth-shaped $\mathbf{p}^{+}$strips.
\end{abstract}

Index Terms-Charge sharing, multi-anode linear drift detectors, sawtooth design, X-ray spectroscopy.

\section{INTRODUCTION}

$\mathbf{O}$ NE-DIMENSIONAL position-sensitive spectroscopy of low-energy X-rays in applications such as X-ray diffraction analysis can be realized by a multi-anode linear silicon drift detector (MLSDD). The position information is obtained only from the $\mathrm{n}^{+}$anode pixel signals. The necessity to detect low-energy X-rays (down to $200 \mathrm{eV}$ ) sets strict limits for the level of electronic noise, which should not exceed $10 \mathrm{rms}$ electrons. Broadening of the electron cloud during its drift toward the anode pixels is a major problem that one has to face when developing an MLSDD. The resulting split events (only part of the signal electrons is collected at one anode pixel) will decrease the achievable energy resolution. This cannot be tolerated for low-energy X-ray detection. We have solved this problem by a nonconventional detector design with sawtooth-shaped $\mathrm{p}^{+}$ strips, referred to as a multi-anode sawtooth silicon drift detector (MSSDD). The sawtooth strips induce potential gutters, forcing electrons to drift towards a single anode pixel without additional lateral broadening [1].

Recently [2], we reported an energy resolution of $450 \mathrm{eV}$ full-width half-maximum (FWHM) for the $5.89 \mathrm{keV}$ line of ${ }^{5} \tilde{\mathrm{F}} \mathrm{Fe}$ at $233 \mathrm{~K}$ with an MSSDD having an anode pitch of $500 \mu \mathrm{m}$. The energy resolution was dominated by the heating of our detector causing a continuous increase of the leakage current. The heating was caused by a high power consumption in the resistor

\footnotetext{
Manuscript received October 13, 2000; revised January 24, 2001. This work was supported by the Netherlands Technology Foundation (STW).

J. Sonský, R. W. Hollander, and C. W. E. van Eijk are with the Radiation Technology Group, Interfaculty Reactor Institute, Delft University of Technology, Delft 2629 JB, The Netherlands (e-mail: sonsky@iri.tudelft.nl).

P. M. Sarro is with Delft Institute of Microelectronics and Submicron Technology, Delft University of Technology, Delft 2628 CD, The Netherlands.

V. Kouchpil is with the Nuclear Physics Institute, Rež 250 68, Czech Republic.

Publisher Item Identifier S0018-9499(01)04777-3.
}

chains (mainly in the external ones). The bulk doping nonuniformities of the wafers were another problem. These local variations of bulk doping concentrations cause perturbations of the applied electric field. Consequently, the distortions of the confining potential gutters resulting in a leak of guided signal electrons [3] and the deviations of the electron trajectories from straight lines [4] are observed. So far, we have used Czochralski wafers (thickness of $500 \mu \mathrm{m}$ ) for detector fabrication. In this type of wafer, the doping nonuniformity was measured to be as high as $15 \%$. Neutron transmutation doped (NTD) wafers having a doping nonuniformity as low as a few percent should be preferentially used to fabricate linear silicon drift detectors.

In this paper, we present X-ray spectroscopy measurements obtained with MSSDDs fabricated on NTD wafers of a thickness of $290 \mu \mathrm{m}$. The problem of the detector heating is solved by a new biasing approach minimizing the number of external connections. We will present results of the performance of an MSSDD with $\mathrm{p}^{+}$strips on both sides of the detector. Eventually, we plan to use an MSSDD with a semicontinuous backplane. This detector with an anode pitch of $500 \mu \mathrm{m}$ was designed and fabricated, but results are not yet available.

\section{Detector Design AND Electron Confinement}

MSSDDs with a total active area of $2.5 \times 1.3 \mathrm{~cm}^{2}$ have been fabricated on 4-in wafers of NTD silicon with a resistivity of 3.5 $\mathrm{k} \Omega \mathrm{cm}$ and a thickness of $290 \mu \mathrm{m}$. The detector is a bidirectional MSSDD with a maximum drift distance of $1.25 \mathrm{~cm}$ in both directions. The anode pitch is $250 \mu \mathrm{m}$, and the $\mathrm{p}^{+}$strip pitch is 200 $\mu \mathrm{m}$. The detector is subdivided into four sections with sawtooth angles $\alpha=0^{\circ}$ (straight strips), $30^{\circ}, 45^{\circ}$, and $60^{\circ}$. A resistor of $200 \mathrm{k} \Omega$ was implanted between adjacent $\mathrm{p}^{+}$strips. The layout of MSSDDs together with detailed description of the design can be found in [1].

To achieve confinement of sufficient electron capacity, it is preferred to design the sawtooth strips on both sides of the detector. In this way, the confining effects, which propagate from the surface, sum and result in a potential minimum at the center of the wafer (see Fig. 1). We have used the SEMISIM package [5] to calculate these potential distributions. We assumed electric fields of $390 \mathrm{~V} / \mathrm{cm}$ (a potential difference of $7.8 \mathrm{~V}$ between adjacent strips) and $280 \mathrm{~V} / \mathrm{cm}$ (a potential difference of $5.6 \mathrm{~V}$ ) on the anode side and p-side of the detector, respectively. These fields correspond to the measurement conditions.

The disadvantage of strips on the p-side (radiation entrance side) of the detector is the positive oxide charge accumulated in the oxide between strips. The positive charge affects the potential distribution beneath the oxide regions close to the surface and creates potential "pocket"-like distributions between 


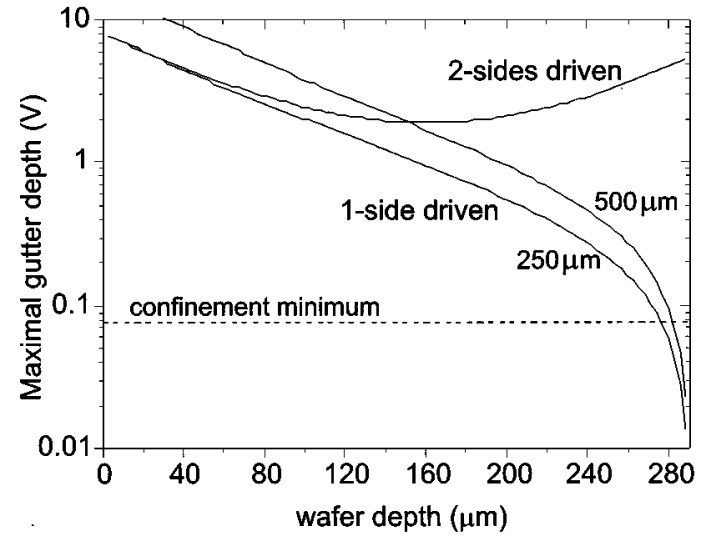

Fig. 1. The maximal depth of potential gutters as a function of the wafer depth supposing that sawtooth strips are placed on both sides (two-side driven confinement, anode pitch of $250 \mu \mathrm{m}$ ) or only on one side (one-side driven, anode pitch of 250 and $500 \mu \mathrm{m}$ ). The other parameters used for calculation are strip pitch of $200 \mu \mathrm{m}$ and $\alpha=60^{\circ}$. The drift fields are $390 \mathrm{~V} / \mathrm{cm}$ (n-side) and $280 \mathrm{~V} / \mathrm{cm}$ (p-side). The horizontal dashed line shows the depth of potential gutters necessary to confine a hypothetical repulsion-free amount of charge.

the strips. The signal electrons generated by low-energy X-ray photons absorbed near the surface will therefore be partially captured, and consequently split events will occur. For this purpose, a shallow continuous $\mathrm{p}^{+}$implantation on the $\mathrm{p}$-side is the ideal solution. However, using continuous implantation on one side of a large detector limits the maximum drift field applicable on the other side. If the potential difference between opposite electrodes across the wafer exceeds the bulk depletion voltage, the drifting potential minimum is set close to the surface and the signal electrons will be lost. As a tradeoff between strips and continuous implantation, we have designed a set of eight wide straight strips on the p-side of the detector. A stair-like potential distribution is applied on these strips. In this way, a reasonably large drift field can be applied on the n-side of the detector. The total area covered with oxide is also minimized. For this type of detector, the confinement is only one-side driven. Nevertheless, the potential gutters (for a drift field of $390 \mathrm{~V} / \mathrm{cm}$ and $\alpha=60^{\circ}$ ) are still deep enough to guarantee the electron confinement supposing that the signal electrons are not drifting closer than about $15 \mu \mathrm{m}$ from the p-side of the detector (see Fig. 1).

\section{EXPERIMENTAL RESULTS}

The detectors were mounted on ceramic boards that allow cooling down to $\sim 200 \mathrm{~K}$. The front-end p-JFETs of the preamplifiers are mounted close to the readout anode pixels to minimize the stray capacitance. The rest of the low-noise preamplifier [6] is placed on the board. The presented results were obtained from sections of the MSSDD with $\alpha=0^{\circ}$ and $60^{\circ}$ with strips on both sides.

A vacuum setup with liquid nitrogen cooling was used to carry out the room- and low-temperature X-ray spectroscopy measurements. We have used an uncollimated ${ }^{5 \tilde{5}} \mathrm{Fe}$ radioactive source for these measurements. The signals are processed by an ORTEC 672 spectroscopy amplifier with a semi-Gaussian filter and fed into an ORTEC AD114. For the light-generated electrons, a pulsed laser beam $(\lambda=675 \mathrm{~nm})$ was used. The intensity of the laser beam was adjusted to produce $\sim 25000$ signal

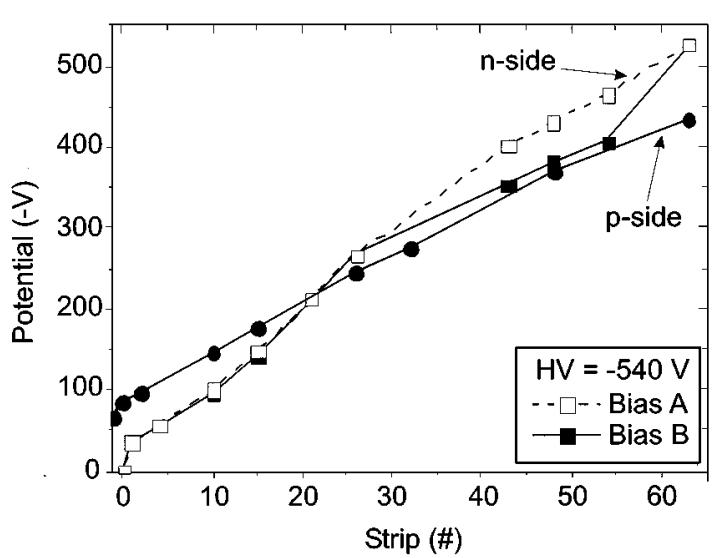

Fig. 2. Measured values of the voltages on certain strips on both sides of the tested MSSDD. We have used a 1-G $\Omega$ probe to measure these voltages.

electrons. An XY micrometric stage allows generation of the signal electrons along the entire active area of the detector. The voltages on $\mathrm{p}^{+}$strips were measured using a $1-\mathrm{G} \Omega$ probe.

\section{A. Biasing}

To minimize the number of external connections to the biasing strips and external voltage dividers, we have used a self-biasing approach [7]. Only a few $\mathrm{p}^{+}$strips are externally biased on the $\mathrm{n}$-side, so a negative potential decreases in approximately uniform steps from the outermost $\mathrm{p}^{+}$strip $\left(\mathrm{n}_{63}\right)$ to the first $\mathrm{p}^{+}$ strip $\left(n_{1}\right)$. The other strips are biased via the implanted resistors. On the opposite p-side, we bias externally only one strip $\left(\mathrm{p}_{0}\right)$ beneath the array of anode pixels. The potential on this strip has to be about $-80 \mathrm{~V}$ in order to focus the drifting electrons toward readout anode pixels. The high-voltage $\left(\mathrm{p}_{63}\right)$ strip is biased by means of the reach-through effect between this strip and the exactly opposite strip $n_{63}$. When the voltage applied on strip $n_{63}$ reaches the bulk depletion voltage, a hole current starts to flow between these two strips. From this point, the voltage on strip $\mathrm{p}_{63}$ follows the applied voltage on strip $\mathrm{n}_{63}$ with a $90-\mathrm{V}$ lag. In Fig. 2, we have plotted voltages measured on different strips on both sides of the detector. We will refer to the biasing described above as bias A.

The disadvantage of this biasing method is a large potential difference between the opposite sides starting from strip number 43. In this region, the drifting potential minimum is positioned very close to the surface and one can expect losses of signal electrons. To improve this, we have set the voltage on strip $n_{54}$ to a value approaching the one measured on the opposite strip $\mathrm{p}_{54}$. In this way, the drifting potential minimum will be located sufficiently far away from the surface up to strip number 54 (see Fig. 2). The rest of the biasing (referred as bias B) remains unchanged. The voltage distribution on the p-side is not influenced by this modification. The average drift field is about $390 \mathrm{~V} / \mathrm{cm}$ for both types of biasing.

\section{B. Laser-Light Response}

The experiment with laser-light-generated electrons is used to verify the confinement effect of the sawtooth strips and the uniformity of the response over the whole active area. Fig. 3 shows a plot of normalized charge collection along the drift length at 


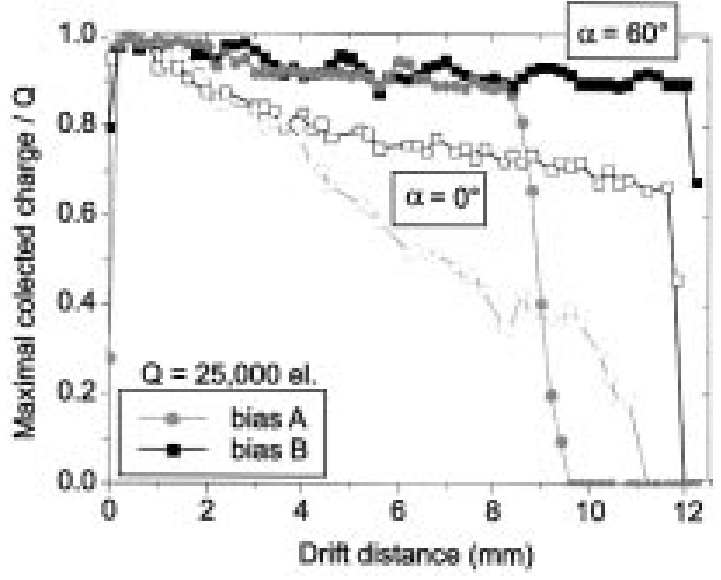

Fig. 3. Normalized charge collection at one anode pixel for $\alpha=0^{\circ}$ and $60^{\circ}$ measured with different biasing. The laser spot was always positioned in the center of the active area belonging to the tested anode pixel.

one anode pixel for $\alpha=0^{\circ}$ and $60^{\circ}$ for biasing $\mathrm{A}$ and $\mathrm{B}$, and charge injection at the center of the active area belonging to the readout anode pixel. It is clearly visible that the signal can be read out only from about $75 \%$ of the total active area when using bias A. The electrons generated in the remaining $~ 25 \%$ of the active area are lost in the surface. The situation dramatically improves when switching to bias B. For $\alpha=60^{\circ}$, we collect more than $93 \%$ of injected charge along the whole drift length, which indicates that also the confinement is well under control. The problems with bulk doping nonuniformities were also solved by using NTD wafers. We have measured straight electron trajectories as expected.

\section{X-Ray Spectroscopy Measurements}

The ${ }^{55} \mathrm{Fe}$ spectra obtained with an MSSDD for $\alpha=0^{\circ}$ and $60^{\circ}$ at low temperature are shown in Fig. 4(a) and (b). During the measurements, the ${ }^{55} \mathrm{Fe}$ source $\left(\sim 1 \times 1 \mathrm{~cm}^{2}\right)$ placed above the center of the detector irradiates approximately the whole active area of the detector. In the case of $\alpha=60^{\circ}$, we have measured an energy resolution of $191 \mathrm{eV} \mathrm{FWHM} \mathrm{(18.1} \mathrm{rms} \mathrm{electrons)} \mathrm{for}$ a shaping time of $6 \mu$ s at $213 \mathrm{~K}$. The split events are almost completely eliminated by using the sawtooth-shaped $\mathrm{p}^{+}$strips. The peak-to-valley ratio is $50: 1$. The observed small background is expected to be due to the edge effects. The energy resolution slightly deteriorates at higher temperatures, and $\mathrm{FWHM}=375$ $\mathrm{eV}$ (42 rms electrons) was measured at room temperature. In the case of $\alpha=0^{\circ}$, despite of a small electronic noise, the spectroscopic quality worsens tremendously due to the high amount of split events.

The results presented above were obtained using a low-noise preamplifier [6], where the feedback capacitor is discharged by a slightly forward-biased gate-to-source junction of a p-channel JFET. A module including a low-noise p-channel JFET and a feedback capacitor (50 fF) was designed and fabricated. The input capacitance of the p-JFET is about $0.5 \mathrm{pF}$. To highlight the influence of major noise components at low temperatures, we have plotted measured equivalent noise charge (ENC) as a function of the shaping time in Fig. 5. We can determine the overall capacitance, leakage current, and $1 / f$ noise by fitting the experimental points [8]. As one can see in Fig. 5, the ENC

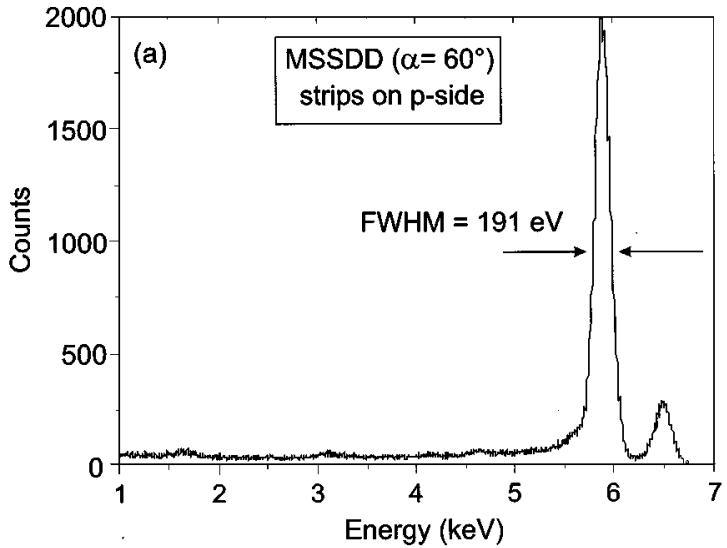

(a)

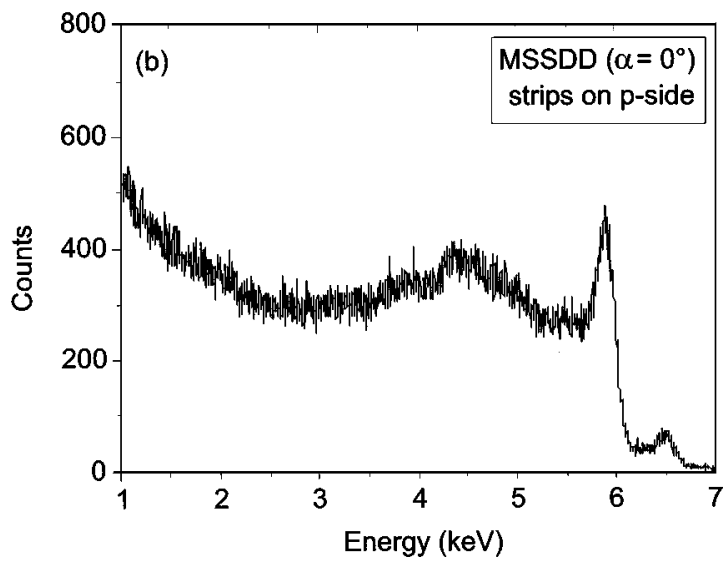

(b)

Fig. 4. Pulse height spectra of ${ }^{55} \mathrm{Fe}$ measured with one anode pixel of MSSDD for (a) $\alpha=60^{\circ}$ and (b) $\alpha=0^{\circ}$ at a temperature of $213 \mathrm{~K}$ with a shaping time of $6 \mu \mathrm{s}$. The detector was operated using bias B.

is dominated by the $1 / f$ noise corresponding to about $15 \mathrm{rms}$ electrons. The $1 / f$ noise of the front-end p-JFET contributes less than one electron to the total ENC [9]. We suspect that a poor $n$-implantation of the readout anode pixels is responsible for this high level of $1 / f$ noise. For this implantation, we use a relatively high dose of arsenic $\left(5 \times 10^{15}\right.$ ions $\left./ \mathrm{cm}^{2}\right)$, and the implanted ions are activated at a temperature of $600{ }^{\circ} \mathrm{C}$. Under these condition, only a small part of the implanted ions is activated and the damaged lattice is probably not entirely recovered. The quality of this junction should be improved by realizing this implantation with phosphorus instead.

The influence of the biasing methods A and B on the spectroscopic quality of the detector is negligible. A small worsening of the energy resolution is observed at room temperature, where the leakage current slightly increases for bias B due to the larger active area. For $\alpha=0^{\circ}$, a small improvement of the spectroscopic quality is observed when using bias B due to improved charge collection (see Fig. 3).

\section{CONCLUSION}

We have presented new results of X-ray spectroscopy measurements on MSSDD with strips on both sides and an anode pitch of $250 \mu \mathrm{m}$ fabricated on NTD wafers. Until now, circular SDD with one central anode was used for high-resolution X-ray 


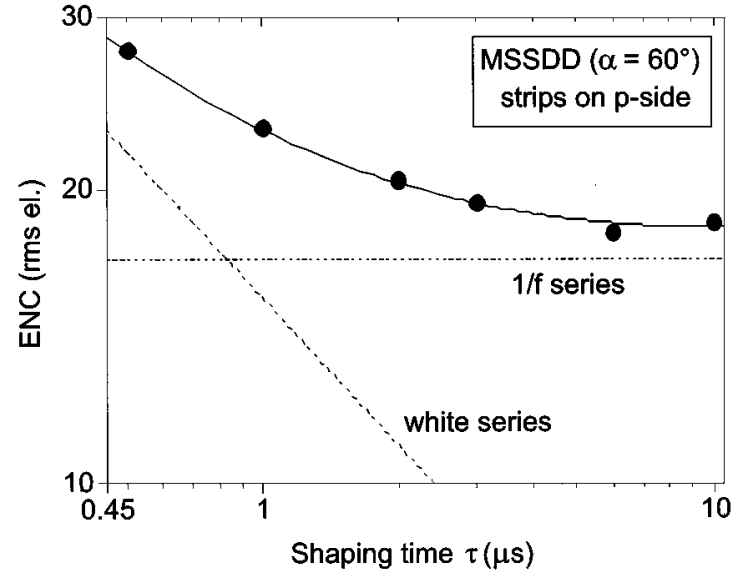

Fig. 5. ENC of the MSSDD $\left(\alpha=60^{\circ}\right)$ readout system as a function of the shaping time. The ENC values were measured at a temperature of $213 \mathrm{~K}$. The most important contribution to the noise is the $1 / f$ series noise and the white series noise coming from the total capacitance.

spectroscopy. We have proven that the spectroscopic quality of our position-sensitive MSSDDs is equal to the quality of a circular SDD. Naturally, the next step is to test an MSSDD with a semicontinuous backplane, which is necessary for detection of low-energy X-ray photons. The performed simulations verified that the charge confinement is satisfactory also in this case. The energy resolution of the MSSDD $\left(\alpha=60^{\circ}\right)$ is 191-eV FWHM per anode pixel at low temperatures. This is practically identical to the best energy resolution measured with a circular SDD fabricated by the same technology and using the same readout. Fur- ther improvement of the energy resolution can be obtained by integrating the front-end JFET into the readout anode pixel and also by improving the quality of the n-implantations.

\section{REFERENCES}

[1] J. Šonský, H. Valk, C. P. Allier, R. W. Hollander, C. W. E. van Eijk, and P. M. Sarro, "Diminished electron cloud broadening in a silicon drift detector by saw tooth $\mathrm{p}^{+}$strips," IEEE Trans. Nucl. Sci., vol. 46, pp. $53-58,1999$.

[2] J. Šonský, J. Huizenga, R. W. Hollander, C. W. E. van Eijk, and P. M. Sarro, "X-ray detection with multi-anode sawtooth silicon drift detectors," IEEE Trans. Nucl. Sci., vol. 47, pp. 750-755, 2000.

[3] J. Sonský, H. Valk, J. Huizenga, R. W. Hollander, C. W. E. van Eijk, and P. M. Sarro, "Electron confinement in multi-anode saw tooth silicon drift detectors with an anode pitch of $250 \mu \mathrm{m}$," IEEE Trans. Nucl. Sci., vol. 46, pp. 271-274, 1999.

[4] A. Castoldi, S. Chinnici, E. Gatti, A. Longoni, M. Sampietro, A. Vacchi, and P. Rehak, "A method for doping fluctuations measurement in high resistivity silicon," J. Appl. Phys., vol. 71, no. 7, pp. 3593-3599, 1992.

[5] E. A. Hijzen, E. M. Schooneveld, C. W. E. van Eijk, and R. W. Hollander, "New silicon drift detector design for diminishing lateral diffusion," Nucl. Instrum. Meth., vol. A335, pp. 271-275, 1993.

[6] G. Bertuccio, P. Rehak, and D. Xi, "A novel charge sensitive preamplifier without the feedback resistor," Nucl. Instrum. Meth., vol. A326, pp. 71-76, 1993.

[7] A. Castoldi, W. Chen, E. Gatti, P. Holl, and P. Rehak, "Fast silicon drift photodiodes free from bias connections on the light entering side," Nucl. Instrum. Meth., vol. A439, pp. 483-496, 2000.

[8] G. Bertuccio and A. Pullia, "A method for the determination of the noise parameters in preamplifying systems for semiconductor radiation detectors," Rev. Sci. Instrum., vol. 64, no. 11, pp. 3294-3298, 1993.

[9] J. Šonský, R. N. Koornneef, L. K. Nanver, G. W. Lubking, J. Huizenga, R. W. Hollander, and C. W. E. van Eijk, "Low Noise p-channel JFET's for X-ray spectroscopy with silicon drift detectors," IEEE Trans. Nucl. Sci., submitted for publication. 